\title{
Improvement of LMCTS Position Accuracy using DR-FNN Controller
}

\author{
Jin Woo Lee*, Jin Ho Suh", Young Jin Lee ${ }^{* *}$, and Kwon Soon Lee ${ }^{* * *}$ \\ * Department of Electrical Engineering, Dong-A University \\ ** Department of Electrical Instrument of Control, Korea Aviation Polytechnic College \\ *** Division of Electrical, Electromics and Computer, Dong-A University
}

\begin{abstract}
Alostract
In this paper, we will introduce a control strategy based on the permanent magnet linear synchronous motor (PMLSM) container transfer system using soft-computing algorithm. Linear motor-based container transport system (LMCTS) is horizontal transfer system for the yard automation, which has been proposed to take the place of automated guided vehicle in the maritime container terminal. LMCTS is considered as that the system is changed its model suddenly and variously by loading and unloading container. The proposed control system is consisted of two DR-FNNs that act the role of controller and system emulator. Consequently, the system had the predictable structure and an ability to adapt for a huge variation of rolling friction, detent force, and sudden changes of its weight by loading and unloading.
\end{abstract}

Key Words : Linear Motor-based Container Transfer System, position control, detent force, friction, DR-FNN

\section{Introduction}

Recently for the port automation, many technologies have been developed. For the yard automation technology, one of them, the well known an automated guided vehicle (AGV) had been proposed. But an AGV system had various problems that included the difficulty of control, complexity, low speed, heavy weight, low position accuracy, etc. Above all, the main problem is the part of navigation that had difficulty to apply in the whole system and to work together with other systems. Recently, LMCTS has been developed to solve these problems. LMCTS is based on the concept of linear motor and rail structure. The main benefits of LMCTS include the high force density and no need for sub-systems. Most importantly, the high precision and accuracy associated with the simplicity in mechanical structure[1]. The system is considered the permanent magnet linear synchronous motor (PMLSM) that is consists of stator modules on the rail and shuttle car. Because of the large variant of mover's weight by the loading and unloading containers, the difference of each characteristic of the stator modules, and a stator module's default etc. LMCTS is able to consider as that the system is changed its model suddenly and variously [2],. Then, we will introduce the control strategy that is a multi-step prediction control for LMCTS using two DR-FNNs.

The proposed control system is used the network that is based on fuzzy neural network. It is composed of the structure of neural network that have the fuzzy inference ability and the

Manuscript received Aug. 20, 2004; revised Sep. 07, 2004. This work was supported by the National Research Laboratory Program of the Korean Ministry of Science \& Technology (MOST). recurrent loops. In addition, it has ability to adjust the structure of network[3,4,7]. Layers in the network are consisted of the adjustable number of nodes which act a role such as the membership function or rule base. We use two units of DR-FNN and each network has two output nodes in the system. One is a predicted system output, another is a predicted reference signal, and others are control input signals from each other network. Then the system has an ability to adapt for the external disturbance, detent force, force ripple, and sudden changes of itself by unifying network weights of controller and emulator.

This paper is organized as follows: Section 2 shows the overview of LMCTS. In Section 3, the proposed control algorithm is described. The computer simulation is conducted in Section 4, followed by the conclusions.

\section{Modeling of LMCTS}

\subsection{System Dynamics}

The system consists of a substructure, shuttle cars, and a control system. The motor style is permanent magnet synchronous linear motor as shown in figure 1. Because of the permanent magnets are installed under the plate of shuttle car, no power cables and other devices are required onto the shuttle car. The considerable shuttle car's dynamic equation is as follow[3,4]:

$$
\ddot{x}=\frac{1}{M}\left(F_{x}-f_{\text {friction }}-f_{d e t}-f_{\text {ripple }}-f_{\text {dis }}-f_{\text {wind }}\right) .
$$

where the various parameters given by (1) are defined by

$F_{x} \quad$ thrust force;

$M \quad$ mass of shuttle car and container 
$x \quad$ shuttle car position;

$f_{\text {fric }}$ friction force;

$f_{\text {ripple }}$ force ripple;

$f_{\text {det }} \quad$ detent force;

$f_{\text {dis }}$ external disturbances;

$f_{\text {wind }}$ wind force.

The major components of force and the relationship between the thrust force, friction, detent force, vertical force, and weight are shown in figure 1 . Here, the system input is the bidirectional force on the same stator module that is composed of the longitudinal force $F_{x}$ and vertical force $F_{z}$, respectively. Especially, the levitation force $F_{z}$ is able to increase or to reduce the shuttle car's mass for the vertical axis only. By these effect, the friction force is changed simultaneously. Therefor it can heighten the acceleration and deceleration performance.

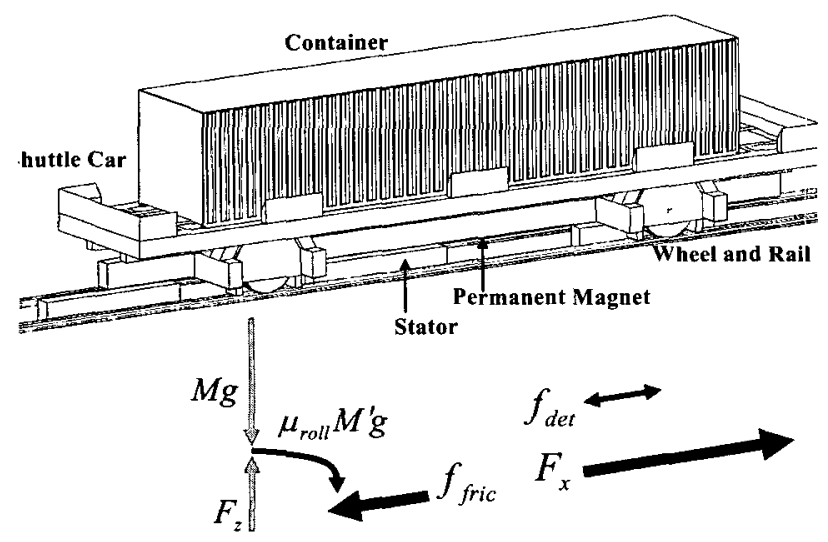

Fig. 1. Configuration and components of LMCTS

The mass of shuttle car is able to modify step function type by loading and unloading container as (2).

$$
M_{\min } \leq M(t) \leq M_{\max }
$$

where, $M_{\min }$ is only the weight of shuttle car ( 8 ton) and $M_{\max }$ is the weight of the loaded shuttle car with heaviest container ( 60 ton).

Because the ripple, wind force, and disturbance term are few the amount than the friction and detent force that are the main problem for the position control, these were included to $f_{e}$ in this paper. Then the considered shuttle car's dynamic equation is described as follow:

$$
F_{x}=M(t) \cdot \dot{x}+f_{\text {fric }}(\dot{x}, \mathbb{M})+f_{\text {det }}+f_{e}
$$

Where, $M$ is considered the changed mass by the levitation force and it influences in the friction force. The thrust force for the longitudinal axis and levitation force for the vertical axis are decided by the design according to a mechanical design of a linear motor as shown by figure 2 , and then these are described as follows[5,6]:

$$
\begin{aligned}
& F_{x}=k_{F 0}(\delta) I_{1} \sin \left(\frac{\pi}{\tau} x\right) \\
& F_{z}=-k_{z S}(\delta) I_{1}{ }^{2}-k_{z M S}(\delta) I_{1} \cos \left(\frac{\pi}{\tau} x\right)
\end{aligned}
$$

where,

$k_{F O}, k_{z S}, k_{z M S}$ coefficients of thrust force and lift forces concerning the structure parameters of motor;

$I_{1}$ effective armature current;

$\tau$ pole pitch;

$\delta \quad$ air-gap length.

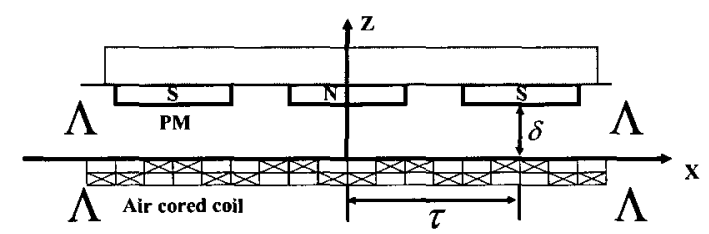

Fig. 2. Part of permanent magnetic synchronous motor

Figure 3 is presented the relationship between the thrust and levitation force. The area (a) and (c) are the proposed area to reduce the effect of friction by Yoshida[5,6]. The area of deceleration is (a) and it is used the positive levitation force. Area (c) is the acceleration area using levitation force with the reduction of friction force.

However, we propose the deceleration area as (b). This area can be used inhalation force with the increase of friction force. Then quick stop characteristics are respected than (a).

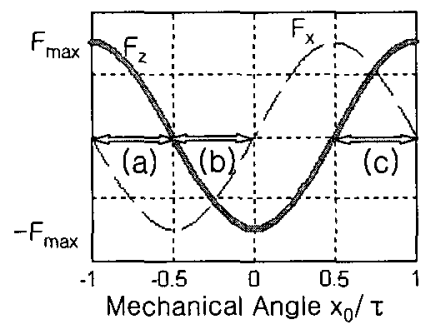

Fig. 3. Relationship between vertical and longitudinal force, and areas for acceleration and deceleration

To consider the friction model, we used the following type equation in $[3,4,11]$ and it is

$$
f_{\text {fric }}=\left[f_{c}+\left(f_{s}-f_{c}\right) e^{-\left(\dot{x} / \dot{x}_{s}\right)^{2}}+f_{v} \dot{x}\right] \operatorname{sign}(\dot{x}) .
$$

where,

$$
\begin{array}{cl}
f_{c} & \text { Coulomb friction; } \\
f_{s} & \text { static friction; } \\
f_{v} & \text { viscous friction; } \\
\bar{x}_{s} & \text { lubricant parameter. }
\end{array}
$$

However it is need to modify the equation that is included the effect of the mass of shuttle car mass for vertical axis by the container loading/unloading and the use of levitation force. 
To consider the change by the force, $\mathbb{T}$ is can be described as (7). By its variation, the changed friction force can be described by ( 8 ).

$$
\begin{aligned}
& M\left(t, F_{z}\right)=M(t)-\mu_{\text {scate }}\left(F_{z} / g\right) \\
& f_{\text {fri }}=\left[\overrightarrow{f_{c}}(M)+\left\{\left(\overrightarrow{f_{s}}(M)-\vec{f}_{d}(M)\right\} e^{\left.-(x) x_{s}\right\}^{z}}\right.\right.
\end{aligned}
$$

where, $\dot{x}_{S}$ is the experimental coefficient of reduction velocity from static friction to Coulomb friction in the stribeck effect. Figure 4 shows the friction model that was considered the range of mass variation is $10 \sim 60$ ton and velocity is -10 $\sim 10 \mathrm{~m} / \mathrm{s}$. In the figure, the area of (A) and (B) is presented the dead zone for the system input. That is, if $F_{x}$ and other longitudinal forces does not get over the boundary surface, these have no influence with the system[12,13]. The included dead zone friction is described as (9).

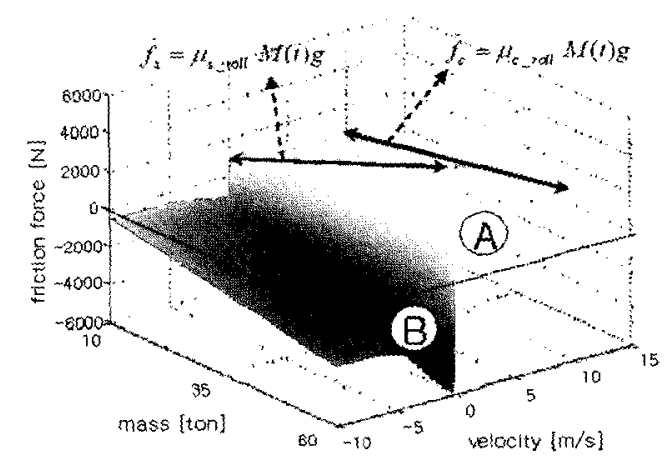

Fig. 4. Friction model of LMCTS with stribeck effect

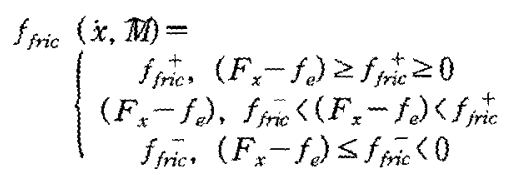

The external disturbance $F_{e}$ is able to consider the white noise as $f_{e}(t, x, \dot{x})$. And the detent force can be described as the function for distance, because it depends on the motor magnet configuration. In this study, because the interval of stator modules equipment are intermittent, it was considered the harmonic sine wave as $(10)$. Where, $K_{1}(\delta)$ and $K_{2}(\delta)$ are the gain that are depended on the air gap, permanent magnet size, etc.

$$
\begin{aligned}
f_{d e t}(x)= & K_{1}(\delta)(35 \sin (2 \pi x / P+\pi / 4) \\
& +K_{2}(\delta) \sin (6 \pi x / P+0.09 \pi)
\end{aligned}
$$

\subsection{LMCTS Driver System}

Figure 5 shows the block-diagram of whole driver system of LMCTS. It is composed of the sensor, input limiter, current coordinate translator, PWM invertor, etc.

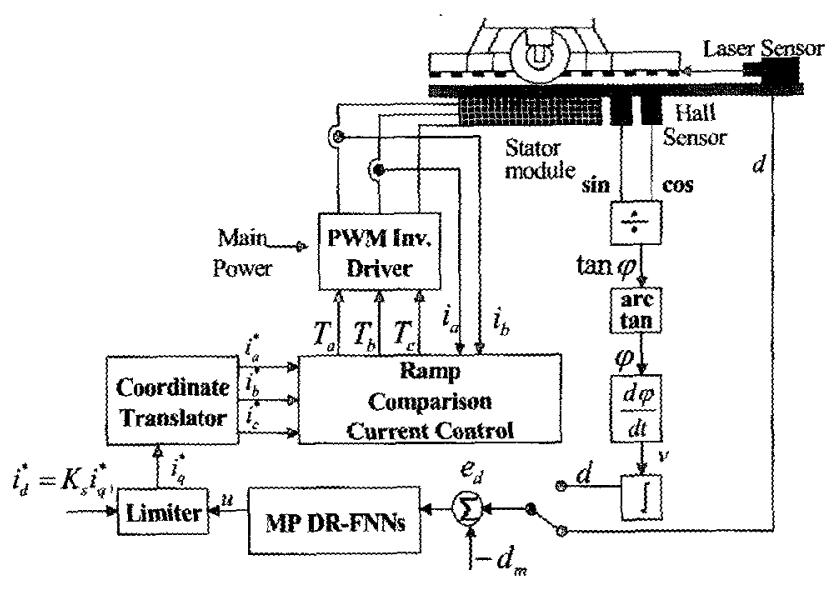

Fig. 5. Block-diagram of LMCTS driver system

LMCTS driver is based on the general PMLSM form as follows $[3,4]$

$$
\begin{aligned}
& v_{q}=R_{s} i_{q}+p \lambda_{q}+\omega_{e} \lambda_{d}, \\
& v_{d}=R_{s} i_{d}+p \lambda_{d}+\omega_{e} \lambda_{q}
\end{aligned}
$$

Flux linkages for each axis, angular velocities, and electric linear velocity are represented by (12) and (13), respectively.

$$
\begin{aligned}
& \lambda_{q}=L_{q} i_{q}, \\
& \lambda_{d}=L_{d} i_{d}+\lambda_{F M} \\
& \omega_{e}=n_{b} \omega_{r}, \\
& \omega_{r}=\pi v / \tau, \\
& v_{e}=n_{p} y=2 \tau f_{e}
\end{aligned}
$$

Electromagnetic power and Electromagnetic force are represented by (14) and (15), respectively.

$$
\begin{aligned}
& P_{e}=F_{e} v_{e}=3 n_{b}\left\{\lambda_{d} i_{q}+\left(L_{d}-L_{q}\right) i_{d^{j_{q}}}\right\} \omega_{q} / 2 \\
& F_{e}=3 \pi n_{p}\left(\lambda_{d_{q}}+\left(L_{d}-L_{q}\right) i_{d^{2}} i_{q}\right) / 2 \tau
\end{aligned}
$$

where,

$\begin{array}{lll}v_{d}, \quad v_{q} & \text { d-q axis voltages; } \\ i_{d}, \quad i_{q} & \text { d }-\mathrm{q} \text { axis currents; } \\ R_{\mathrm{s}} & \quad \text { phase winding resistance; } \\ L_{d,}, L_{\psi} & \text { d-q axis inductances; } \\ \omega_{r} & \text { angular velocity of the mover; } \\ \omega_{e} & \text { electrical angular velocity; } \\ \lambda_{P M} & \text { permanent magnet flux linkage; } \\ n_{p} & \text { number of primary poles; } \\ p & \text { differential operator; } \\ v & \text { linear velocity of the mover; } \\ x & \text { distance (mover's position); } \\ \tau & \text { pole pitch; } \\ v_{e} & \text { electric linear velocity; } \\ f_{e} & \text { electric frequency. }\end{array}$

The simplified PMLSM driver and its gain are describe as 
(16) and (17) respectively. And $i_{d}^{*}$, the levitation force input current, is configured as (18).

$$
\begin{aligned}
& F_{e}=K_{F} i_{q}^{*} \\
& K_{F}=3 \pi n_{p} \lambda_{P M} / 2 \tau \\
& i_{d}^{*}=K_{s} \cdot i_{q}^{*}
\end{aligned}
$$

\section{Control System Design}

In this paper, the structure of network is composed of having two input and output nodes, respectively. In the proposed control system, two networks are used to the controller and emulator. Figure 6 shows DR-FNN that is used the emulator unit.

One of the output nodes is prediction of the plant output for the one-step ahead, and the other is the predicted control input $u$. And inputs are recurrent delayed values of control input and system output about p-step time index backward. The network can be considered the recurrent style. Because outputs are used inputs these are delayed in next time index.

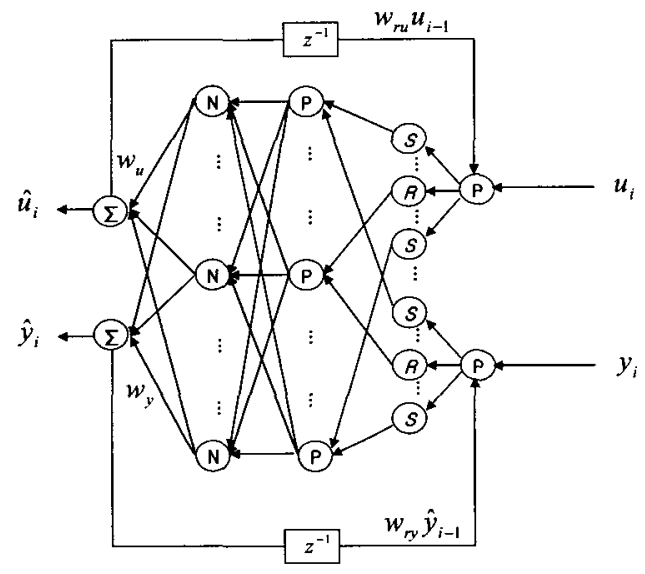

Fig. 6. DR-FNN unit

In figure 6, Layer 1 is the input layer for the linguistic variables. Layer 2 consists of the membership function that has nodes based on the radial basis function. In the case of $j=1$ and $u$, for two nodes of edged side in layer 2 , the membership function get the shape of sigmoid function, and these are optimized automatically by the back-propagation method. The output of membership function is presented by equation (19) in the second layer [7, 8]

$$
\begin{aligned}
& \mu_{i j}\left(x_{i}\right)=\exp \left\{-\frac{\left(x_{i}-c_{i j}\right)^{2}}{\sigma_{i j}^{2}}\right\} \\
& (i=1,2, \quad j=1,2, \cdots, u)
\end{aligned}
$$

where, ... is the product between the current input and delayed output. and are presented by (20).

$$
\begin{aligned}
& x_{1}(t)=u(t) \cdot w_{r u} \cdot \hat{u}(t-1) \\
& x_{2}(t)=y(t) \cdot w_{r u} \cdot \hat{y}(t-1)
\end{aligned}
$$

Third layer is IF-part for fuzzy rules. For the $\mathrm{j}$-th rule $R_{j}$, its output is described by equation (21) in third layer. The normalized output of third layer was calculated in layer 4 . It can be expressed by (22).

$$
\begin{aligned}
& R_{k}=\exp \left\{-\frac{\sum_{i=1}^{2 p}\left(x_{i}-c_{i j}\right)^{2}}{\sum_{i=1}^{2 p} \sigma_{i j}^{2}}\right\} \\
& N_{k}=R_{k} / \sum_{i=0}^{N} R_{l}
\end{aligned}
$$

The fifth (output) layer computes the output of consequence part. Control input and predicted plant output are calculated by (23) and (24) respectively.

$$
\begin{aligned}
& \hat{y}(t)=\sum_{k=1}^{N} w_{y k} \cdot N_{k} \\
& \hat{u}(t)=\sum_{k=1}^{N} w_{u k} \cdot N_{k}
\end{aligned}
$$

For the prediction and adaptation, DR-FNN is constructed beside plant as an emulator style. The proposed control system is used two DR-FNNs as shown by figure 7 .

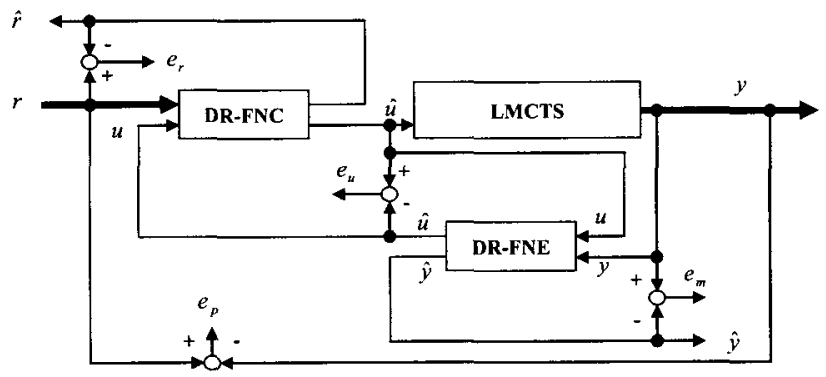

Fig. 7. Control system with controller and emulator

It performs not only controller but also act of on-line identifier by two outputs from network. The system can obtain predictions of reference input signal $\hat{y}_{d}$ and the other control input $\hat{u}$. Then, the system has two control inputs protruded from controller and emulator. In figure 8 , the system structure has four errors from networks and plant. Errors and network learning is can be described as follows:

$$
\left.\begin{array}{l}
e_{p}=r(t)-y(t), E_{p}=\frac{1}{2} e_{p}^{2} \\
w_{l, k}(t+1)=w_{l, k}(t)+\eta\left\{-\frac{\partial E_{p}}{\partial w_{l, k}}\right\}
\end{array}\right\}
$$


The predicted reference input signal $\hat{y}_{d}$ and the predicted plant output $\hat{y}$ are trained by $e_{r}$ and $e_{d}$, respectively. To improve the adaptability in this case, the proposed control scheme is regarded as the predicted next step system input because two units of DR-FNN are used to a controller and emulator as shown in figure 3 .

In the part of controller, if DR-FNC were trained enough by the various patterns of reference input signals, network can simulate without the plant virtually using predicted next reference input. Even though network was trained well for some known patterns also, input is the value that can not be guarantee absolutely. And the reference input can be decided by unknown pattern.

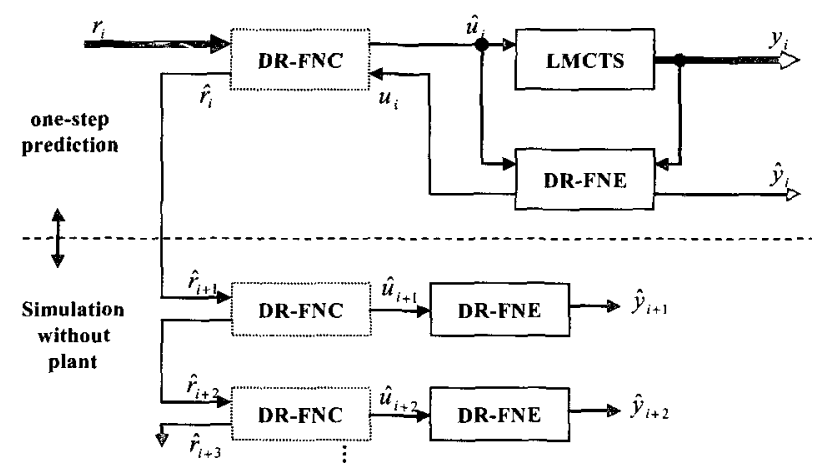

Fig. 8. System structure for multi-step prediction

To decide the number of nodes and prediction step, an objective function is needed as the proposed (29).

To the system is stable, there is need to minimize the $Q$ and to make the gradient of $Q$ to the negative value. Therefore we preconfigured the threshold values $\Delta Q_{\min }$ and $\Delta Q_{\max }$, and configured the following rules. In case of (30), the nodes of network are added and the prediction step is reduced. In case of (31), the network keeps its structure. The case of (32) is the number of network nodes is reduced and the prediction step must be increased.

$$
Q=\sqrt{\alpha E_{p}^{2}+\beta E_{u}^{2}+\gamma E_{r}^{2}+\delta E_{m}^{2}}
$$

- Case 1:

$$
\text { IF } \frac{\partial Q(t)}{\partial t} \geq \Delta Q_{\max }
$$

THEN increase node and reduce prediction step.

- Case 2:

$$
\text { IF } \Delta Q_{\max } \leq \frac{\partial Q(t)}{\partial t}<\Delta Q_{\min }
$$

THEN keep the structure of network. (31)

- Case 3:

$$
\text { IF } \frac{\partial Q(t)}{\partial t}<\Delta Q_{\min } \approx 0
$$

THEN decrease node and extend prediction step

\section{Simmalation and Resulls}

Figure 9 and 10 are the response of open loop system for the test thrust force that type is the half sine wave and its maximum force are $130[\mathrm{kN}]$ and $13[\mathrm{kN}]$ respectively. Figure 10 shows that the effective region is only from 2[sec] to $4[\mathrm{sec}]$ for test input and external force, because of the static rolling friction.

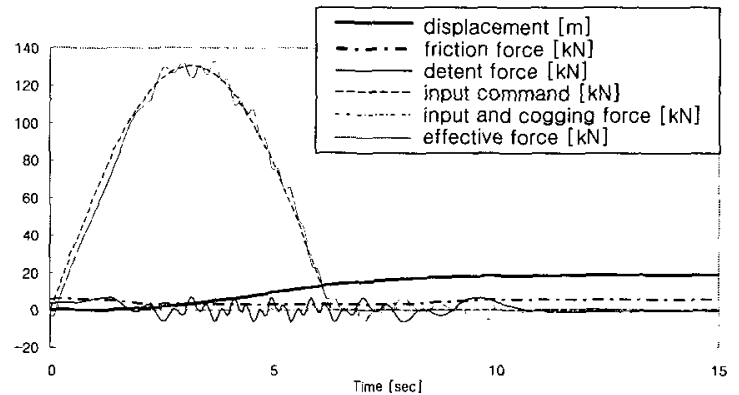

Fig. 9. Open loop system characteristics on the input of half sine wave of maximum $130[\mathrm{kN}]$

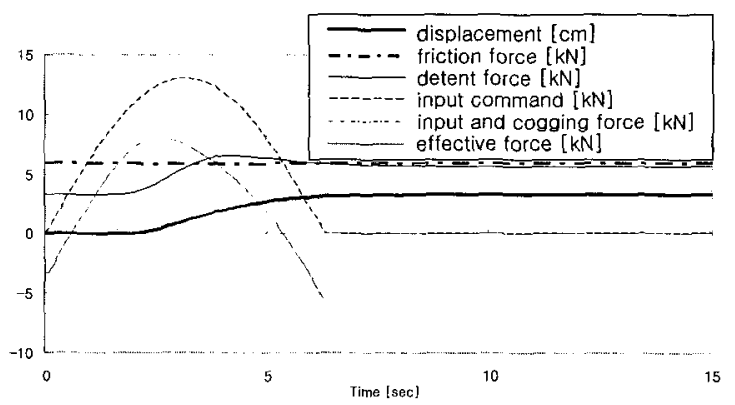

Fig 10. Minuteness motion characteristics on the input of half sine wave of maximum $13[\mathrm{kN}]$

In the simulation, the scenario was composed of the following. At first, the empty shuttle car goes until the point of $33 \mathrm{~m}$ and comes again. Secondly, the container of 40ton is loaded at $50 \mathrm{sec}$. Finally, the loaded shuttle car returns until $55 \mathrm{~m}$ as shown in figure 11 .

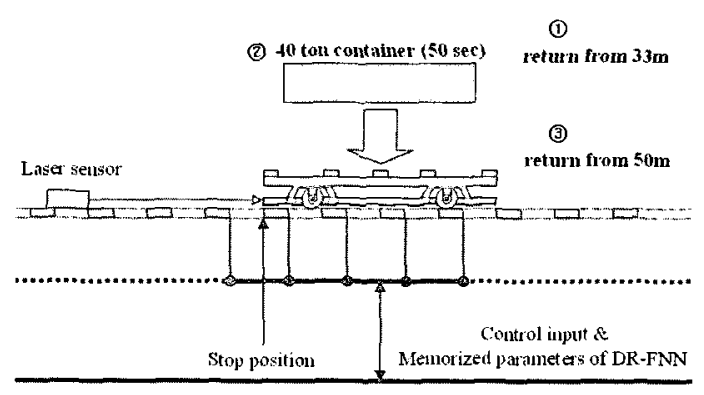

Fig. 11. Positioning System using the method of segment 
In figure 12 , the results of position variation are presented, and the dotted line is a reference and a solid line is a change of the distance. Figure 12(a) is the result for the PID controller was tuned by $\mathrm{ZN}$ method adding trial and error, (b) is for FNN controller was used fixed nodes and its structure, and (c) is the result for the R-FNN that is the recurrent type with the delayed input of network for the position and the velocity respectively. Finally, (d) is presented the result for the proposed control system with separating to 16 node start from 5 membership functions. According as going from (a) to (d), it is improved the response characteristics and reinforced steep weight change of the system and effect of detent force and stator module. Figure 13 shows the variation of distance error for the whole range. The maximum position error is less than $1.8 \mathrm{~cm}$ and the average is about $0.8 \mathrm{~cm}$ in the stop area. Figure 14 and 15 show the used longitudinal and vertical input, and figure 16 is presented the energy consumption by integrating the scaled RMS value of inputs.

(a)

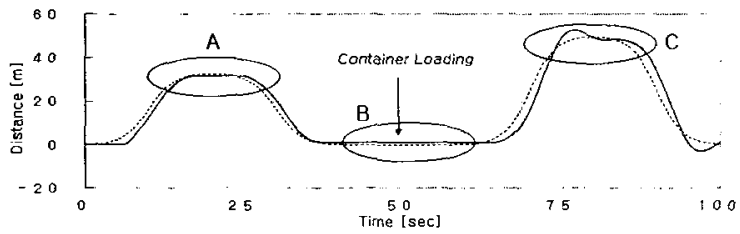

(b)

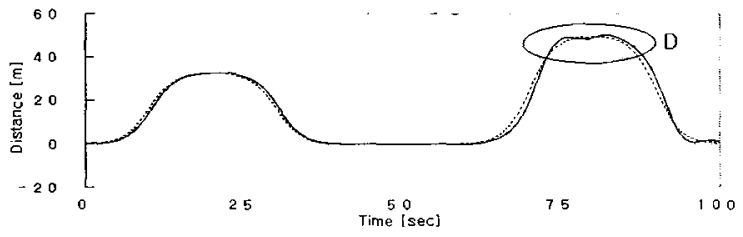

(c)

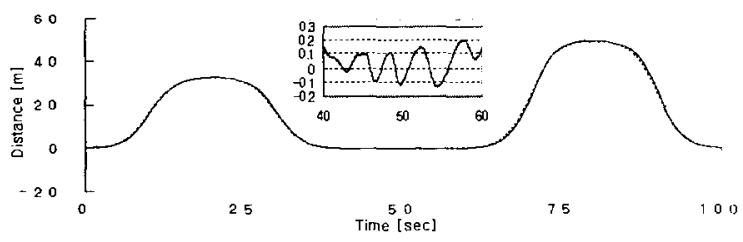

(d)

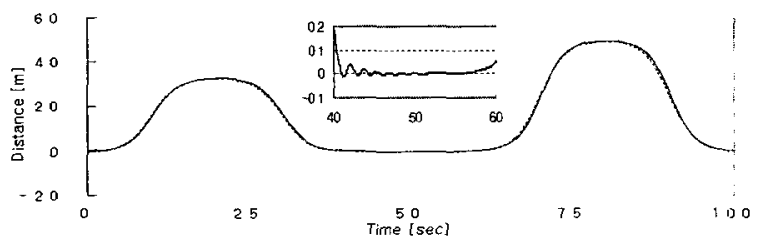

Fig. 12. The reference trajectory and position of shuttle car, (a) PID controller, (b) general fuzzy neural network with fixed parameters, (c) recurrent fuzzy neural network, (d) proposed method

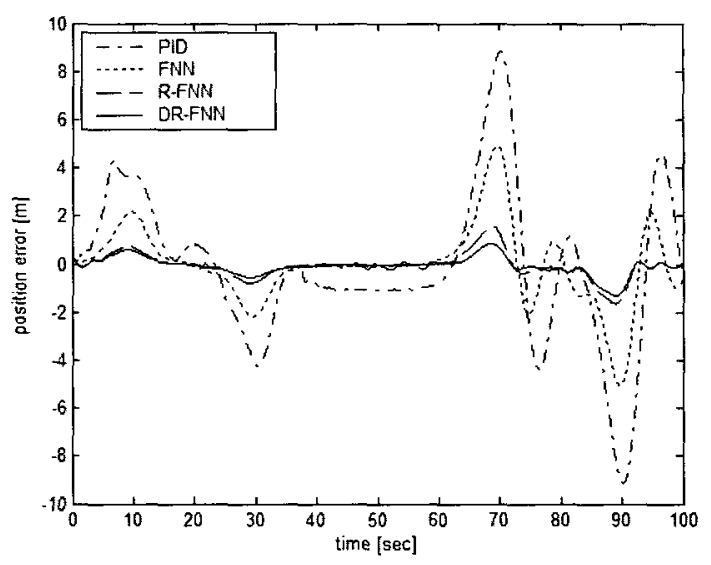

Fig. 13. The variation of position error

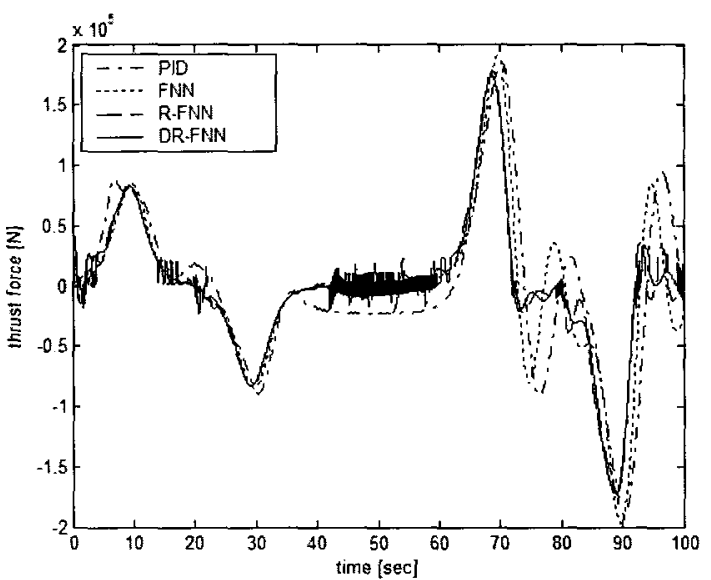

Fig. 14. The variation of thrust force

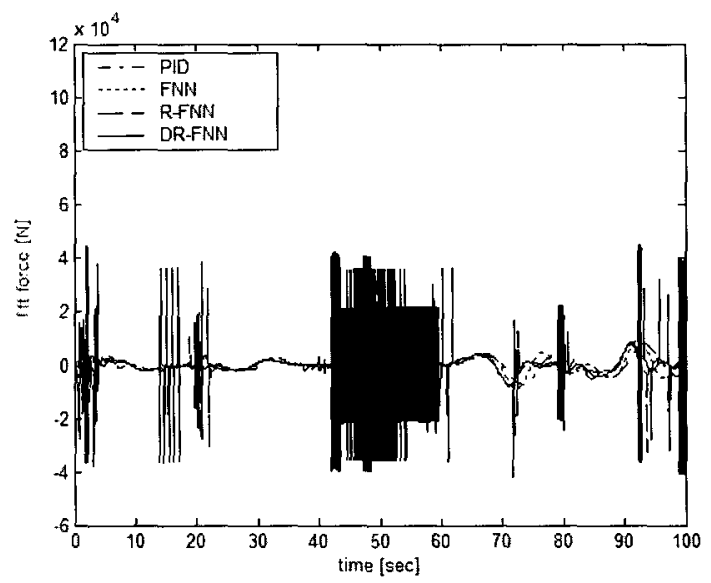

Fig. 15. The variation of vertical force 


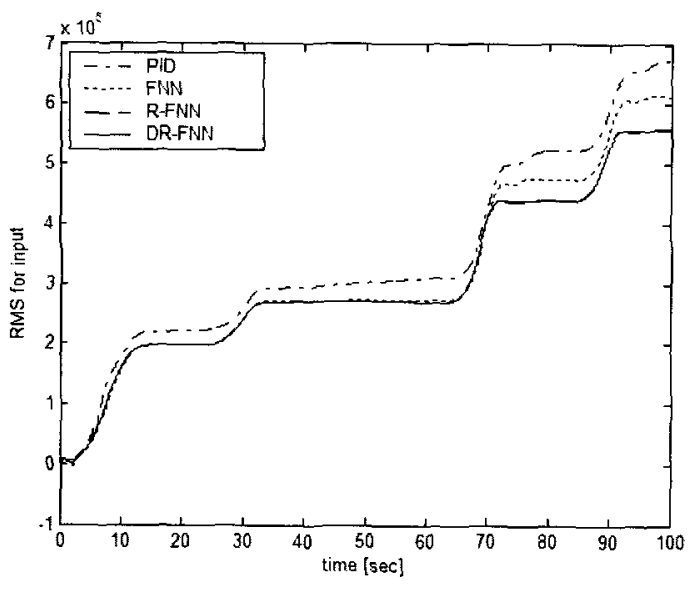

Fig. 16. The variation of vertical force

\section{Conclusion}

In this study, we modeled LMCTS system by modifying the general PMLSM and adding with the considerable various disturbances. In LMCTS, the system included various kinds of problems to control such as the detent force, force ripple, external disturbances, the variation of mover weight, the periodic lack of thrust force, etc. To improve the positioning accuracy and reduce the energy consumption, we proposed a control system that has multi-step predictable structure using two DR-FNN units. In the case of using DR-FNN, there are improvements of $81 \%, 68 \%$, and $3 \%$ than the PID controller, the supervised FNN, and R-FNN, respectively in position accuracy. By multi-step predictable structure, the amount of control input was reduced about $24.6 \%, 8.3 \%, 0.8 \%$ than PID controller, FNN, and R-FNN, respectively.

\section{References}

[1] Klaus-Peter Franke, "Boosting Efficiency of Split Marine Container Terminals by Innovative Technology," IEEE Intelligent Transportation Systems Conference Proc., Oakland, USA, August, pp. 774-779, 2001.

[2] K. K. Tan, S. N. Huang, and T. H. Lee, "Robust Adaptive Numerical Compensation for Friction and Force Ripple in Permanent-Magnet Linear Motors," IEEE Trans. on Magnetics, Vol. 38, No. 1, pp. 221-228, 2002.

[3] F. J. Lin, R. J. Wai, and C. M. Hong, "Hybrid Supervisory Control Using Recurrent Fuzzy Neural Network for Tracking Periodic Inputs," IEEE Trans. on Neural Networks, Vol. 12, No. 1, January, 2002.

[4] Rong-Jong Wai, Faa-Jeng Lin, "Adaptive RecurrentNeural-Network Control for Linear Induction Motor," IEEE Trans. on Aerospace and Electronic Systems, Vol. 37, No. 4, pp. 1176-1192, 2001.
[5] K. Yoshida, H. Takami, X. Kong, and A. Sonoda, "Mass Reduction and Propulsion Control for a PermanentMagnet Linear Synchronous Motor Vehicle," IEEE Trans. on Industry Applications, Vol. 37, No. 1, pp. 67-72, 2001.

[6] K. Yoshida, X. Kong, and H. Takami, "Network Transport Automation Study of PM LSM Vehicle on Orthogonally-Switching-Guideway," Industry Applications Conference, Vol. 2, pp. 1109-1114, 2000.

[7] Y. Frayman and L. Wang, "A Dynamically-constructed Fuzzy Neural Controller for Direct Model Reference Adaptive Control of Multi-input-multi-output Nonlinear," Soft Computing, Vol. 6, pp. 244-253, 2002.

[8] B. Widrow and E. Walach, Adaptive Inverse Control, Upper Saddle River, Prentice Hall, 1996.

[9] V. Kecman, L. Vlacic, and R. Salman, "Learning in and performance of the new neural network based adaptive backthrough control structure," Proceedings of the 14-th IFAC Triennial World Congress, Beijing, PR Chaina, Vol. K, pp. 133-140, 1999.

[10] T. L. Seng, M. B. Khalid, and R. Y. Yusof, "Tuning of a Neuro-Fuzzy Controller by Genetic Algorithm," IEEE Trans. on Systems, Man, and Cybernetics, Part B: Cybernetics, Vol. 29, No. 2, pp. 226-236, 1999.

[11] Brian Armstrong-Helouvry, Pierre Dupont, and Carlos Canudas De Wits, "A Survey of Models, Analysis Tools and Compensation Methods for the Control of Machines with Friction," Áutomatica, Vol. 30, No. 7. pp. 1083-1138, 1994.

[12] Young H. Kim and Frank L. Lewis, "Neural Network Output Feedback Control of Robot Manipulators," IEEE Trans. on Robotics and Automation, Vol. 15, No. 2, pp. 301-309, 1999.

[13] Young Ho Kim and Frank L. Lewis, "Reinforcement Adaptive Learning Neural-Net-Based Friction Compensation control for High Speed and Precision," IEEE Trans. on Control Systems Technology, Vol. 8, No. 1, pp. 118-126, 2000.

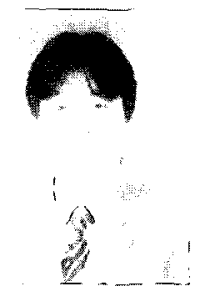

\section{Jin Woo Lee}

received the B.S. degree from Kyungnam University, Masan, and M.S. degree from Department of Electrical Engineering from Dong-A University, Busan, Korea, in 1998 and 2000 , respectively.

$\mathrm{He}$ is studying for the Ph.D. degree in National Research Laboratory(NLR) iPort, Dong-A University, Korea. His recent research interests are intelligent control, soft-computing, and application techniques for port automation system, etc.

$\begin{array}{ll}\text { Phone } & :+82-51-200-6950 \\ \text { Fax } & :+82-51-200-7743 \\ \text { E-mail } & : \text { jinwoo@donga.ac.kr }\end{array}$




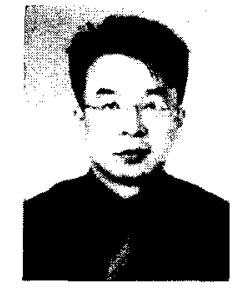

\section{Jin Ho Suh}

received B.S. degree in mathematics from Hanyang University and M.S. degree in control engineering from Pukyong National University in 1993 and 1998, and Ph.D. degree in control engineering from Tokyo Institute of Technology, Japan, in 2002 respectively. He is currently Post-Doctor in

National Research Laboratory(NLR), Dong-A University, Korea. His research interests are nonlinear control algorithm, robotics, and application techniques for port automation system, etc.

Phone : $+82-51-200-6950$

Fax : +82-51-200-7743

E-mail : suhgang@hanmail.net

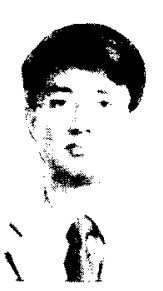

\section{Young Jin Lee}

received B.S., M.S., and Ph.D. degree in Department of Electrical Engineering from Dong-A University, Busan, Korea, in 1992, 1994, and 2000, respectively. Since 2000 , he has been with Department of Electrical Instrument and Control at Korea Aviation Polytechnic College, where he is currently an assistant professor. His research interests are intelligent control, immune algorithm, port automation system.

$\begin{array}{ll}\text { Phone } & :+82-55-830-3482 \\ \text { Fax } & :+82-51-830-3489 \\ \text { E-mail } & : \text { airlee@kopo.or.kr }\end{array}$

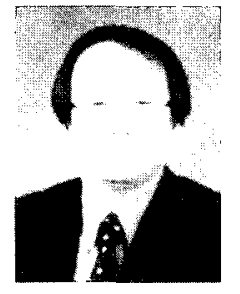

Kwon Soon Lee

received the B.S. degree from Chung Nam National University, Chung-nam, and M.S. degree from Department of Electrical Engineering from Seoul National University, Seoul, Korea, in 1973 and 1979, respectively. He received the $\mathrm{Ph} . \mathrm{D}$. degree from Department of Electrical and Computer Engineering in Oregon State University, USA, in 1990.

Since 1982, he has been with Division of Electrical, Electronic, and Computer in Dong-A University, Busan, Korea. where he is currently professor.

His recent research interests are intelligent control theory, immune algorithm, and application techniques for port automation system, etc.

\author{
Phone : +82-51-200-7739 \\ Fax : +82-51-200-7743 \\ E-mail : kslee@daunet.donga.ac.kr
}

